\title{
On Maximal and Minimal Solutions for Set-Valued Differential Equations with Feedback Control
}

\author{
Ngo Van Hoa and Nguyen Dinh Phu \\ Faculty of Mathematics and Computer Science, University of Science, Ho Chi Minh City, Vietnam \\ Correspondence should be addressed to Ngo Van Hoa, ngovanhoa_clt@yahoo.com \\ Received 11 September 2011; Accepted 8 November 2011 \\ Academic Editor: Ibrahim Sadek
}

Copyright (C) 2012 N. V. Hoa and N. D. Phu. This is an open access article distributed under the Creative Commons Attribution License, which permits unrestricted use, distribution, and reproduction in any medium, provided the original work is properly cited.

In this paper, we present the existence of extremal solutions of set-valued differential equations with feedback control on semilinear Hausdorff space under Hukuhara derivative which is developed under the form $D_{H} X(t)=F(t, X(t), H(t, X(t))), X(0)=X_{0}$, for all $t \in[0, T]$ with the monotone iterative technique and we will verify that monotone sequence of approximate solutions converging uniformly to the solution of the problem, that is useful for optimization problems.

\section{Introduction}

Recently, the study of set differential equations was initiated in a metric space and some basic results of interest were obtained. Some interesting results in this direction have been obtained in a series of works of Professor V. Lakshmikantham and other authors (see [1-5]). Professor V. Lakshmikantham and the other authors considered set differential equations (SDEs) and had some important results on existence, comparison, and stability criteria for SDEs:

$$
D_{H} X(t)=F(t, X(t)),
$$

where $X\left(t_{0}\right)=X_{0}, X(t) \in K_{C C}\left(R^{n}\right)$, and $t \in\left[t_{0}, T\right]=I \subset R_{+}$.

Based on these results, the authors gave the concept of set-valued control differential equation and studied existence and comparison of its solutions (see [6]). In this paper, we investigate an existence result of Peano's type and then consider the existence of extremal solution of set-valued control differential equations. For this purpose, one needs to introduce a partial order in $\left(K_{c}\left(R^{n}\right), D\right)$, prove the required comparison result for strict inequalities, and then, utilizing it, discuss the existence of extremal solutions.

This paper is organized as follows: in Section 2, we recall some basic concepts and notations which are useful in next sections. In Section 3, we present on the existence of 
extremal solutions for SSDEs on semilinear Hausdorff space with the monotone iterative technique and we will verify that monotone sequence of approximate solutions converging uniformly to the solution of the problem.

\section{Preliminaries}

We recall some notations and concepts presented in detail in recent series works of Professor V. Lakshmikantham et al. (see [1]). Let $K_{C C}\left(R^{n}\right)$ denote the collection of all nonempty compact convex subsets of $R^{n}$. Given $A, B \in K_{C C}\left(R^{n}\right)$, the Hausdorff distance between $A$ and $B$ is defined by

$$
D[A, B]=\max \left\{\sup _{a \in A} \inf _{b \in B}\|a-b\|_{R^{n}} \sup _{b \in B} \inf _{a \in A}\|b-a\|_{R^{n}}\right\},
$$

where $\|\cdot\|_{R^{n}}$ denotes the Euclidean norm in $R^{n}$ and $\left\{\theta^{n}\right\}$ 一the zero points set in $K_{C C}\left(R^{n}\right)$. It is known that $\left(K_{C C}\left(R^{n}\right), D\right)$ is a complete metric space and $K_{C C}\left(R^{n}\right)$ is a complete and separable with respect to metric $D$.

We define the magnitude of a nonempty subset of $A$ :

$$
D\left[A, \theta^{n}\right]=\|A\|=\sup \left\{\|a\|_{R^{n}}, a \in A\right\},
$$

where $\theta^{n}$ is the zero element of $R^{n}$ which is regarded as a one point set. $\|A\|=D\left[A, \theta^{n}\right]$-norm in $K_{C C}\left(R^{n}\right)$ is finite when the supremum in (2.2) is attained with $A \in K_{C C}\left(R^{n}\right)$.

The Hausdorff metric (1.1) satisfies the properties below:

$$
\begin{gathered}
D[A+C, B+C]=D[A, B], \quad D[A, B]=D[B, A], \\
D[\lambda A, \lambda B]=\lambda D[A, B], \\
D[A, B] \leq D[A, C]+D[C, B], \\
D\left[A+A^{\prime}, B+B^{\prime}\right] \leq D[A, B]+D\left[A^{\prime}, B^{\prime}\right]
\end{gathered}
$$

for all $A, B, C, A^{\prime}, B^{\prime} \in K_{C C}\left(R^{n}\right)$ and $\lambda \in R_{+}$. If $\alpha, \beta \in R$, and $A, B \in K_{C C}\left(R^{n}\right)$, then

$$
\alpha(A+B)=\alpha A+\alpha B, \quad \alpha(\beta A)=(\alpha \beta) A, \quad 1 \cdot A=A .
$$

It is known that $\left(K_{C C}\left(R^{n}\right), D\right)$ is a complete metric space and if the space $K_{C C}\left(R^{n}\right)$ is equipped with the natural algebraic operations of addition and nonnegative scalar multiplication, then $K_{C C}\left(R^{n}\right)$ becomes a semilinear metric space which can be embedded as a complete cone into a corresponding Banach space.

Let $A, B \in K_{C C}\left(R^{n}\right)$. The set $C \in K_{C C}\left(R^{n}\right)$ satisfying $A=B+C$ is called the Hausdorff difference (the geometric difference) of the sets $A$ and $B$ and is denoted by the symbol $A-B$. 
Given an interval $I$ in $R_{+}$. We say that the set mapping $F: I \rightarrow K_{C C}\left(R^{n}\right)$ has a Hukuhara derivative $D_{H} F\left(t_{0}\right)$ at a point $t_{0} \in I$, if

$$
\lim _{h \rightarrow 0^{+}} h^{-1}\left[F\left(t_{0}+h\right)-F\left(t_{0}\right)\right], \quad \lim _{h \rightarrow 0^{+}} h^{-1}\left[F\left(t_{0}\right)-F\left(t_{0}-h\right)\right]
$$

exist in the topology of $K_{C C}\left(R^{n}\right)$ and are equal to $D_{H} F\left(t_{0}\right)$.

By embedding $K_{C C}\left(R^{n}\right)$ as a complete cone in a corresponding Banach space and taking into account result on the differentiation of Bochner integral, we find that if

$$
F(t)=X_{0}+\int_{t_{0}}^{t} \Phi(s) d s, \quad X_{0} \in K_{C C}\left(R^{n}\right),
$$

where $\Phi: I \rightarrow K_{C C}\left(R^{n}\right)$ is integrable in the sense of Bochner, then $D_{H} F(t)$ exists and the equality $D_{H} F(t)=\Phi(t)$ a.e on $I$ holds.

The Hukuhara integral of $F$ is given by

$$
\int_{I} F(s) d s=\mathrm{cl}\left[\int_{I} f(s) d s: f \text { is a continuous selector of } F\right]
$$

for any compact set $I \subset R_{+}$.

Some properties of the Hukuhara integral are in [1]. If $F: I \rightarrow K_{C C}\left(R^{n}\right)$ is integrable, one has

$$
\begin{gathered}
\int_{t_{0}}^{t_{2}} F(s) d s=\int_{t_{0}}^{t_{1}} F(s) d s+\int_{t_{1}}^{t_{2}} F(s) d s, \quad t_{0} \geq t_{1} \geq t_{2}, \\
\int_{t_{0}}^{t} \lambda F(s) d s=\lambda \int_{t_{0}}^{t} F(s) d s, \quad \lambda \in R .
\end{gathered}
$$

If $F, G: I \rightarrow K_{C C}\left(R^{n}\right)$ are integrable, then $D[F(\cdot), G(\cdot)]: I \rightarrow R$ is integrable and

$$
D\left[\int_{t_{0}}^{t} F(s) d s, \int_{t_{0}}^{t} G(s) d s\right] \leq \int_{t_{0}}^{t} D[F(s), G(s)] d s .
$$

\section{Main Results}

We consider the set-valued differential equations (SSDEs) with feedback control under the form

$$
D_{H} X(t)=F(t, X(t), H(t, X(t))), \quad X(0)=X_{0}, \quad \forall t \in[0, T],
$$

where $F:[0, T] \times K_{C C}\left(R^{n}\right) \times K_{C C}\left(R^{d}\right) \rightarrow K_{C C}\left(R^{n}\right)$ and $H:[0, T] \times K_{C C}\left(R^{n}\right) \rightarrow K_{C C}\left(R^{d}\right)$ is a feedback control, state set $X_{0}, X(t) \in K_{C C}\left(R^{n}\right)$. 
Definition 3.1. The mapping set $X:[0, T] \rightarrow K_{C C}\left(R^{n}\right)$ is called to be a solution of (3.1) on $[0, T]$ if and only if the following conditions are satisfied:

(i) $X(t) \in C^{1}\left[[0, T], K_{C C}\left(R^{n}\right)\right]$ with Hukuhara derivative $D_{H}$ by $t$;

(ii) $X(0)=X_{0}$;

(iii) $F(t, X(t), H(t, X(t))$ is integrable on $[0, T]$;

(iv) for all $t \in[0, T]$,

$$
X(t)=X_{0}+\int_{0}^{t} F(S, X(s), H(s, X(s))) d s
$$

the integral in (3.2) is Hukuhara integral.

In this section, we will use the monotone iterative technique to solve the minimal and maximal solutions of (3.1). To construct the set monotone sequence, we first introduce the following definition.

\section{Definition 3.2. We denote}

(i) by $K^{+}\left(K^{0,+}\right)$ the subfamily of $K_{C C}\left(R^{n}\right)$ consisting of sets $X \in K_{C C}\left(R^{n}\right)$ such that any $x \in X$ is a nonnegative (positive) vector of $n$ components satisfying $x_{i} \geq 0\left(x_{i}>0\right)$ for $i=1,2, \ldots, n$,

(ii) by $K^{-}\left(K^{0,-}\right)$ the subfamily of $K_{C C}\left(R^{n}\right)$ consisting of sets $X \in K_{C C}\left(R^{n}\right)$ such that any $x \in X$ is a nonpostive (negative) vector of $n$ components satisfying $x_{i} \leq 0\left(x_{i}<0\right)$ for $i=1,2, \ldots, n$.

By Definition 3.2, we notice that $K^{+}$is a positive cone in $K_{C C}\left(R^{n}\right)$ and $K^{0,+}$ is the nonempty interior of $K^{+} . K^{-}$is a negative cone in $K_{C C}\left(R^{n}\right)$ and $K^{0,-}$ is the nonempty interior of $K^{-}$. We can therefore induce a partial odering in $K_{C C}\left(R^{n}\right)$. Thus, if $X \in K^{+}\left(K^{0,+}\right)$ is $X \geq \theta^{n}\left(X>\theta^{n}\right)$, that is, with any $x \in X$ is satisfying $x_{i} \geq 0\left(x_{i}>0\right)$ for $i=1,2, \ldots, n$ and $X \in K^{-}\left(K^{0,-}\right)$ is $X \leq \theta^{n}\left(X<\theta^{n}\right)$, that is, with any $x \in X$ is satisfying $x_{i} \leq 0\left(x_{i}<0\right)$ for $i=1,2, \ldots, n$. Now we define the ordering in $K_{C C}\left(R^{n}\right)$.

Definition 3.3. For any $X, Y \in K_{C C}\left(R^{n}\right)$, if there exists a $Z \in K_{C C}\left(R^{n}\right)$ such that $Z \in K^{+}\left(K^{0,+}\right)$ and $X=Y+Z$, then we write $X \geq Y(X>Y)$. Similarly, if there exists a $\bar{Z} \in K_{C C}\left(R^{n}\right)$ such that $\bar{Z} \in K^{-}\left(K^{0,-}\right)$ and $X=Y+\bar{Z}$, then we write $X \leq Y(X<Y)$.

Theorem 3.4. Assume the following:

(H1) $F(\cdot, X(\cdot), U(\cdot)) \in C\left[[0, T], K_{C C}\left(R^{n}\right)\right]$ is monotone nondecreasing in $(X, U) \in$ $K_{C C}\left(R^{n}\right) \times K_{C C}\left(R^{d}\right)$ for every $t \in[0, T]$, that is, for fixed $\left.t \in[0, T], F\left(t, X_{1}, U_{1}\right)\right) \geq$ $F\left(t, X_{2}, U_{2}\right)$ wherever $X_{1} \geq X_{2}, U_{1} \geq U_{2}$ and $H(\cdot, X(\cdot)) \in C\left[[0, T], K_{C C}\left(R^{n}\right)\right]$ is monotone nondecreasing in $X \in K_{C C}\left(R^{n}\right)$ for every $t \in[0, T]$, that is, for fixed $t \in[0, T]$, $H\left(t, X_{1}\right) \geq H\left(t, X_{2}\right)$ wherever $X_{1} \geq X_{2}$;

(H2) there exist $V, W \in C^{1}\left[I, K_{C C}\left(R^{n}\right)\right]$ such that $D_{H} V(t) \leq F(t, V(t), H(t, V(t)))$ and

$$
D_{H} W(t) \geq F(t, W(t), H(t, W(t)))
$$


(H3) for any $X_{1}, X_{2} \in K_{C C}\left(R^{n}\right)$ with $X_{1} \geq X_{2}$ and some positive number real $L>0$ such that

$$
F\left(t, X_{1}, H\left(t, X_{1}\right)\right) \leq F\left(t, X_{2}, H\left(t, X_{2}\right)\right)+L\left(X_{1}-X_{2}\right)
$$

then $V(t) \leq W(t)$ for $t \in I$ provided $V(0) \leq W(0)$.

Proof. For any $\eta=\left(\eta_{1}, \eta_{2}, \ldots, \eta_{n}\right)>\theta^{n}$, we define $W_{\eta}(t)=W(t)+\eta e^{2 L t}$ and we note that $V(0) \leq W(0) \leq W_{\eta}(0)$. By using (2.5), we infer $D_{H} W_{\eta}(t)=D_{H} W(t)+2 L \eta e^{2 L t}$. Let $t_{\delta}$ be the supremum of all positive number $\delta$ such that $V(0)<W_{\eta}(0)$ implies $V(t)<W_{\eta}(t)$ on $[0, \delta]$. Thus $t_{\delta}>0$ and $V\left(t_{\delta}\right)<W_{\eta}\left(t_{\delta}\right)$. Using $(\mathrm{H} 1)-(\mathrm{H} 3)$, we get

$$
\begin{aligned}
D_{H} V\left(t_{\delta}\right) \leq F\left(t_{\delta}, V\left(t_{\delta}\right), H\left(t_{\delta}, V\left(t_{\delta}\right)\right)\right) & \leq F\left(t_{\delta}, W_{\eta}\left(t_{\delta}\right), H\left(t_{\delta}, W_{\eta}\left(t_{\delta}\right)\right)\right) \\
& \leq F\left(t_{\delta}, W\left(t_{\delta}\right), H\left(t_{\delta}, W\left(t_{\delta}\right)\right)\right)+L \cdot\left(W_{\eta}\left(t_{\delta}\right)-W\left(t_{\delta}\right)\right) \\
& \leq F\left(t_{\delta}, W\left(t_{\delta}\right), H\left(t_{\delta}, W\left(t_{\delta}\right)\right)\right)+L \eta e^{2 L t_{\delta}} \\
& \leq D_{H} W\left(t_{\delta}\right)+2 L \eta e^{2 L t_{\delta}}=D_{H} W_{\eta}\left(t_{\delta}\right) .
\end{aligned}
$$

Equation (2.5), together with (3.5), implies that there exists an $h>0$ such that

$$
W_{\eta}\left(t_{\delta}\right)-V\left(t_{\delta}\right)<W_{\eta}(t)-V(t), \quad t_{\delta}<t<t_{\delta}+h
$$

This contradicts that $t_{\delta}>0$ is the supremum in view of the continuity of function involved and consequently that the inequality $V(t)<W_{\eta}(t)$ holds for $t \in I$. Taking the limit $\eta \rightarrow 0$ yields the desired result. This proof is complete.

Corollary 3.5. Let $V, W \in C^{1}\left[I, K_{C C}\left(R^{n}\right)\right]$ such that $D_{H} V(t) \leq D_{H} W(t)$ for all $t \in I$, then $V(0) \leq$ $W(0)$ implies that $V(t) \leq W(t)$ for all $t \in I$.

Proof. It is clear from the proof of Theorem 3.4.

Definition 3.6. $V, W \in C^{1}\left[I, K_{C C}\left(R^{n}\right)\right]$ are said to be the lower solution and upper solution of (3.1) respectively if

$$
\begin{gathered}
D_{H} V(t) \leq F(t, V(t), H(t, V(t))), \quad V(0) \leq X(0), \\
D_{H} W(t) \geq F(t, W(t), H(t, W(t))), \quad W(0) \geq X(0) .
\end{gathered}
$$

Theorem 3.7 (existence of solution). Assume $V, W \in C^{1}\left[I, K_{C C}\left(R^{n}\right)\right]$ are lower solution and upper solution of (3.1), respectively, and assumptions (H1), (H3) are satisfied, then there exists solution $X(t)$ of (3.1). 
Proof. For any $\eta=\left(\eta_{1}, \eta_{2}, \ldots, \eta_{n}\right)>\theta^{n}$, we define $W_{\eta}(t)=W(t)+\eta e^{2 L t}$ and $V_{\eta}(t)+\eta e^{2 L t}=V(t)$. Let $t_{\delta}$ be the supremum of all positive number $\delta$ such that $V(0)<W_{\eta}(0)$ implies $V(t)<W_{\eta}(t)$ on $[0, \eta]$. Thus $t_{\delta}>0$ and $V\left(t_{\delta}\right)<W_{\eta}\left(t_{\delta}\right)$, by putting the above we infer that

$$
W_{\eta}\left(t_{\delta}\right)=W\left(t_{\delta}\right)+\eta e^{2 L\left(t_{\delta}\right)}>W\left(t_{\delta}\right), \quad W_{\eta}(0)=W(0)+\eta>W(0)
$$

Similarly, $V_{\eta}\left(t_{\delta}\right)<V\left(t_{\delta}\right)$ and $W_{\eta}(0)<V(0)$. By using Theorem 3.4, we have $V_{\eta}\left(t_{\delta}\right)<V\left(t_{\delta}\right)<$ $W\left(t_{\delta}\right)<W_{\eta}\left(t_{\delta}\right)$. Since $V, W$ are lower and upper solutions of (3.1), we have that

$$
V_{\eta}(0)<V(0)<X(0)<W(0)<W_{\eta}(0), \quad V_{\eta}\left(t_{\delta}\right)<X\left(t_{\delta}\right)<W_{\eta}\left(t_{\delta}\right)
$$

where $X\left(t_{\delta}\right)$ is solution of (3.1). Now, we wish to show that $V_{\eta}(t)<X(t)<W_{\eta}(t)$ on $I$. If it is not true, then there exists a $t_{1} \in I$ such that $X\left(t_{1}\right)=W_{\eta}\left(t_{1}\right)$ and $V_{\eta}(t)<X(t)<W_{\eta}(t)$ on $I \backslash\left\{t_{1}\right\}$. This implies that $X\left(t_{1}\right)=W_{\eta}\left(t_{1}\right)>W\left(t_{1}\right)$ and $D_{H} X\left(t_{1}\right)>D_{H} W\left(t_{1}\right)$. Equation (2.5), together with $D_{H} X\left(t_{1}\right)>D_{H} W\left(t_{1}\right)$, implies that there exists an $h>0$ such that

$$
X\left(t_{1}\right)-W\left(t_{1}\right)<X(t)-W(t), \quad t_{\delta}<t_{1}<t_{\delta}+h .
$$

This contradicts that $X\left(t_{1}\right)>W\left(t_{1}\right)$, hence we have that $X(t)<W_{\eta}(t)$. Similarly, we can show that $V_{\eta}(t)<X(t)$ and hence relation $V_{\eta}(t)<X(t)<W_{\eta}(t)$ holds for all $t \in I$. Now as $\eta \rightarrow 0$, we conclude that $V(t) \leq X(t) \leq W(t)$. The proof is complete.

Definition 3.8. Let $\underline{M}(t), \bar{M}(t)$ are said to be minimal and maximal solutions of (3.1), respectively, if they both are solution of (3.1) and satisfy $\underline{M}(t) \leq X(t) \leq \bar{M}(t)$ for every solution $X(t)$ of (3.1) with $V(t) \leq X(t) \leq W(t)$ for all $t \in I$, where $V, W$ are the lower and upper solutions of (3.1) respectively with $V(t) \leq W(t)$ for all $t \in I$.

Theorem 3.9. Assume that

(M1) equation (3.1) has the lower solution $V$ and upper solution $W$ with $V, W \in$ $C^{1}\left[I, K_{C C}\left(R^{n}\right)\right]$ and $V(t) \leq W(t)$ for all $t \in I ;$

(M2) hypotheses (H1), (H3) satisfy;

(M3) $F$ is map bounded sets into bounded sets in $K_{C C}\left(R^{n}\right)$.

Then there exists monotone sequence $\left\{W^{n}(t)\right\}$ and $\left\{V^{n}(t)\right\}$ in $K_{C C}\left(R^{n}\right)$ such that $V^{n}(t) \rightarrow \underline{M}(t)$, $W^{n}(t) \rightarrow \bar{M}(t)$ as $n \rightarrow \infty$ in $K_{C C}\left(R^{n}\right)$, where $\underline{M}(t), \bar{M}(t)$ are the minimal and maximal solutions of (3.1), respectively.

Proof. Let us construct the set of integrodifferential sequences by

$$
\begin{gathered}
V^{n+1}(t)=X_{0}+\int_{0}^{t} F\left(s, V^{n}(s), H\left(s, V^{n}(s)\right)\right)(0.1), \\
W^{n+1}(t)=X_{0}+\int_{0}^{t} F\left(s, W^{n}(s), H\left(s, W^{n}(s)\right)\right) d s(0.2)
\end{gathered}
$$


for $n=0,1,2, \ldots$, we prescribe $V^{0}(t)=V(t)$ and $W^{0}(t)=W(t)$, for all $t \in I$. From (2.5), (3.1) and using Definition 3.1 we get

$$
\begin{aligned}
& D_{H} V^{n+1}(t)=F\left(t, V^{n}(t), H\left(t, V^{n}(t)\right)\right), \quad V^{n+1}(0)=X_{0}(0.4), \\
& D_{H} W^{n+1}(t)=F\left(t, W^{n}(t), H\left(t, W^{n}(t)\right)\right), \quad W^{n+1}(0)=X_{0}(0.5) \text {. }
\end{aligned}
$$

First, we claim that the iterations are such that

$$
V^{0} \leq V^{1} \leq \cdots \leq V^{n} \leq W^{n} \leq \cdots \leq W^{1} \leq W^{0}
$$

Now we show that $V^{0}(t) \leq V^{1}(t) \leq W^{1}(t) \leq W^{0}(t)$. Consequently, we have to show that (i) $V^{0}(t) \leq V^{1}(t)$, (ii) $W^{1}(t) \leq W^{0}(t)$ and (iii) $V^{1}(t) \leq W^{1}(t)$. By using Definition 3.6 and (3.11), (3.12), then (i) is proved. Indeed, by $V^{0}(t)=V(t)$ is a lower solution of (3.1) and following Definition 3.6 we get $V^{0}(0) \leq X_{0}=V^{1}(0)$, addition

$$
D_{H} V^{1}(t)=F\left(t, V^{0}(t), H\left(t, V^{0}(t)\right)\right), \quad D_{H} V^{0}(t) \leq F\left(t, V^{0}(t), H\left(t, V^{0}(t)\right)\right)
$$

Hence $D_{H} V^{1}(t) \geq D_{H} V^{0}(t)$ and using Corollary 3.5 we infer $V^{0}(t) \leq V^{1}(t)$ for all $t \in I$. Similarly, we use Definition 3.6 and (3.11), (3.12), then (ii) is proved. Using (M1), we get

$$
D_{H} V^{1}(t)=F\left(t, V^{0}(t), H\left(t, V^{0}(t)\right)\right) \leq F\left(t, W^{0}(t), H\left(t, W^{0}(t)\right)\right)=D_{H} W^{1}(t),
$$

addition $V^{1}(0)=X_{0}=W^{1}(0)$ and Corollary 3.5, then (iii) is proved.

By using inductive method, we assume $V^{0} \leq V^{n-1} \leq V^{n} \leq W^{n} \leq W^{n-1} \leq W^{0}$ on $I$, then we have to claim that $V^{n} \leq V^{n+1} \leq W^{n+1} \leq W^{n}$, by means of the monotone property of $F, G$ we obtain

$$
\begin{gathered}
D_{H} V^{n}(t)=F\left(t, V^{n-1}(t), H\left(t, V^{n-1}(t)\right)\right) \leq F\left(t, V^{n}(t), H\left(t, V^{n}(t)\right)\right)=D_{H} V^{n+1}(t), \\
D_{H} W^{n}(t)=F\left(t, W^{n-1}(t), H\left(t, W^{n-1}(t)\right)\right) \geq F\left(t, W^{n}(t), H\left(t, W^{n}(t)\right)\right)=D_{H} W^{n+1}(t) .
\end{gathered}
$$

From $V^{n}(0)=V^{n+1}(0)=W^{n+1}(0)=W^{n}(0)=X_{0}$, and by virtue of Corollary 3.5 we get $V^{n} \leq V^{n+1}$ and $W^{n+1} \leq W^{n}$ for all $t \in I$. Again, by means of the monotone property of $F, G$ and our assumption, we have

$$
D_{H} V^{n+1}(t)=F\left(t, V^{n}(t), H\left(t, V^{n}(t)\right)\right) \leq F\left(t, W^{n}(t), H\left(t, W^{n}(t)\right)\right)=D_{H} W^{n+1}(t),
$$

for all $t \in I$. Using again Corollary 3.5, we get $V^{n+1} \leq W^{n+1}$. Consequently,

$$
V^{0} \leq V^{1} \leq \cdots \leq V^{n} \leq W^{n} \leq \cdots \leq W^{1} \leq W^{0} .
$$

Combining (3.11) and $F$ is continuous multiplication, it follows that $V^{n}, W^{n}$ are continuous for $n=0,1,2, \ldots$. 
Now using the corresponding of (3.11) and the properties of the Hausdorff metric and the Hukuhara integral, together with the assumption (M3), we prove the equicontinuity of the sequences $\left\{V^{n}\right\}$ and $\left\{W^{n}\right\}$ below. Consider for any $t<t_{h}, t, t_{h} \in I$, we have

$$
D\left[V^{n}\left(t_{h}\right), V^{n}(t)\right] \leq D\left[\int_{t}^{t_{h}} F\left(s, V^{n}(s), H\left(t, V^{n}(t)\right)\right) d s, \theta^{n}\right] \leq M\left(t_{h}-t\right) .
$$

Hence $\left\{V^{n}\right\}$ and $\left\{W^{n}\right\}$ are uniformly bounded and equicontinuity on I. On using AscoliArzela theorem (see [1]) in this setup, we obtain a subsequence $\left\{V^{n_{k}}\right\}$ which converges uniformly to $\underline{M}(t) \in K_{C C}\left(R^{n}\right)$ on $I$. Arguing in a similarly to the $\left\{V^{n}\right\}$, we conclude that $\left\{W^{n}\right\}$ converges uniformly to $\bar{M}(t)$ on $I$. Next, we again consider (3.12), (3.18), respectively, and by using the convergence properties we infer that

$$
\begin{aligned}
D_{H} \bar{M}(t) & =F(t, \bar{M}(t), H(t, \bar{M}(t))), \quad \bar{M}(0)=X_{0}, \\
D_{H} \underline{M}(t) & =F(t, \underline{M}(t), H(t, \underline{M}(t))), \quad \underline{M}(0)=X_{0} .
\end{aligned}
$$

Moreover, by means of (3.18) we easily get $V^{0}(t) \leq \underline{M}(t) \leq \bar{M}(t) \leq W^{0}(t)$ on $I$.

Finally, we show that $\underline{M}(t)$ and $\bar{M}(t)$ are the minimal and maximal solutions of (3.1), respectively. Let $X(t)$ be any solution of (3.1) such that $V^{0}(t) \leq X(t) \leq W^{0}(t)$ for all $t \in I$ and $V^{0}(0) \leq X_{0} \leq W^{0}(0)$ and we need to prove that $V^{0}(t) \leq \underline{M}(t) \leq X(t) \leq \bar{M}(t) \leq W^{0}(t)$ on $I$. Suppose that for some $n, V^{n} \leq X(t) \leq W^{n}$ on $I$. By using monotone nondecreasing of $F, G$, we get

$$
D_{H} X(t)=F(t, X(t), H(t, X(t))) \geq F\left(t, V^{n}(t), H\left(t, V^{n}(t)\right)\right)=D_{H} V^{n+1}(t),
$$

where $X_{0}=V^{n+1}(0)$. Applying Corollary 3.5, then we get $X(t) \leq V^{n+1}(t)$ on $I$ for all $n$. Similarly, we get $V^{n+1} \leq X(t)$ for all $n$. By using assumption $V^{0}(t) \leq X(t) \leq W^{0}(t)$ from the principle of mathematical induction, we infer that $V^{n} \leq X(t) \leq W^{n}$ for all $n$. Taking limit as $n \rightarrow \infty$, then we obtain $\underline{M}(t) \leq X(t) \leq \bar{M}(t)$. The proof is complete.

Corollary 3.10. If addition to the assumptions of Theorem 3.7 assume that $F$ satisfies

$$
F\left(t, X_{1}, H\left(t, X_{1}\right)\right)+L_{1}\left(X_{1}-X_{2}\right) \geq F\left(t, X_{2}, H\left(t, X_{2}\right)\right)
$$

for $X_{1} \geq X_{2} \in K_{C C}\left(R^{n}\right)$ and $L_{1}>0$, then $\underline{M}(t)=X(t)=\bar{M}(t)$ is the unique solution of (3.1).

Example 3.11. We consider set-valued differential equation with feedback control in $K_{C C}(\mathbb{R})$ :

$$
D_{H} X(t)=3 X(t)+\sin (t) X(t), \quad X(0)=[1,2], t \in\left[0, \frac{\pi}{2}\right]
$$

where $F(t, X(t), U(t))=3 X(t)+\sin (t) X(t)$ with $H(t, X(t))=\sin (t) X(t)$ is a contraction feedback. 
We see that $F(t, X(t), H(t, X(t)))$ satisfies (M1)-(M3). Now, we show that (3.23) exits as extremal solutions on $[0, \pi / 2]$. We prescribe $V(t)=X_{0} e^{3 t}-\varepsilon, W(t)=X_{0} e^{4 t}+\varepsilon$ are lower and upper solutions of (3.23) for all $\varepsilon>0$. We note that $D_{H} V(t) \leq 3 V(t)+\sin (t) V(t)$ and $D_{H} W(t) \geq 3 W(t)+\sin (t) W(t)$. Next, let us construct the set sequences by

$$
\begin{aligned}
D_{H} V^{n+1}(t) & =3 V^{n}(t)-\frac{\varepsilon}{n}, \quad V^{n+1}(0)=X_{0}-\frac{\varepsilon}{n}, \\
D_{H} W^{n+1}(t) & =4 W^{n}(t)+\frac{\varepsilon}{n}, \quad W^{n+1}(0)=X_{0}+\frac{\varepsilon}{n},
\end{aligned}
$$

for all $n=0,1,2, \ldots$ we verify that monotone sequences of constructions above such that

(a) $\left\{V^{n}(t)\right\} \stackrel{n \rightarrow \infty}{\longrightarrow} \underline{M}(t)$ and $\underline{M}(t)$ is a minimal of (3.23);

(b) $\left\{W^{n}(t)\right\} \stackrel{n \rightarrow \infty}{\longrightarrow} \bar{M}(t)$ and $\bar{M}(t)$ is a maximal of (3.23).

First, we prove (a). Indeed, let $0<\varepsilon_{2}<\varepsilon_{1} \leq \varepsilon$, then for each positive integer $n$, we consider

$$
\begin{gathered}
D_{H} W^{n+1}(t)=4 W^{n}(t)+\frac{\varepsilon}{n}, \quad W^{n+1}(0)=X_{0}+\frac{\varepsilon}{n}, \\
W(t)=X_{0} e^{4 t}+\varepsilon .
\end{gathered}
$$

Because $W^{n+1}\left(0, \varepsilon_{2}\right) \leq W^{n+1}\left(0, \varepsilon_{1}\right)$ and $D_{H} W^{n+1}\left(t, \varepsilon_{2}\right) \leq 4 W^{n}(t)+\varepsilon_{2} / n$, otherwise $D_{H} W^{n+1}\left(t, \varepsilon_{1}\right) \geq 4 W^{n}(t)+\varepsilon_{2} / n$, hence $D_{H} W^{n+1}\left(t, \varepsilon_{1}\right) \geq D_{H} W^{n+1}\left(t, \varepsilon_{2}\right)$. By using Corollary 3.5, to get $W^{n+1}\left(t, \varepsilon_{2}\right) \leq W^{n+1}\left(t, \varepsilon_{1}\right)$ for all $t \in[0, \pi / 2]$ and $\varepsilon_{2}<\varepsilon_{1}$. On the other hand $W^{n+1}(t) \leq$ $W^{n}(t)$ with $\varepsilon$ fixed.

Since the family of functions $\left\{W^{n+1}(t, \varepsilon)\right\}$ is equicontinuous and uniformly bounded on $[0, \pi / 2]$, it follows Ascoli-Arzela theorem (see [4]) that there exists a decreasing sequence $\left\{\varepsilon /(n+1)_{k}\right\} \stackrel{k \rightarrow \infty}{\longrightarrow} 0$ and uniform limit $\bar{M}(t)=\lim _{k \rightarrow \infty} W^{(n+1)_{k}}(t, \varepsilon)$ exits on $[0, \pi / 2]$. Obviously $\bar{M}(t)=X_{0} \cdot e^{4 t}$, the uniform continuity of $F$ implies that $F\left(t, W^{(n+1)_{k}}(t, \varepsilon), H\left(t, W^{(n+1)_{k}}(t, \varepsilon)\right)\right)$ tends uniformly to $F(t, \bar{M}(t), H(t, \bar{M}(t)))$ as $k \rightarrow \infty$, and thus

$$
W^{(n+1)_{k}}(t, \varepsilon)=X_{0}+\frac{\varepsilon}{n_{k}}+\int_{0}^{\pi / 2}\left(4 W^{(n+1)_{k}}(s, \varepsilon)\right) d s
$$

which in turn yields that the limit $\bar{M}(t)$ is a solution of (3.23) on [0, $\pi / 2]$.

Next we will show that $\bar{M}(t)$ is a required maximal solution of $(3.23)$ on $[0, \pi / 2]$. For this purpose, we observe that $X(0)=X_{0}<X_{0}+\varepsilon / n=W^{n+1}(0, \varepsilon)$ and $F$ is nondecreasing, hence we get

$$
D_{H} X(t)<3 X(t)+\sin (t) X(t)+\frac{\varepsilon}{n} \leq 3 W^{n}(t, \varepsilon)+\sin (t) W^{n}(t, \varepsilon)+\frac{\varepsilon}{n} \leq D_{H} W^{n+1}(t, \varepsilon) .
$$




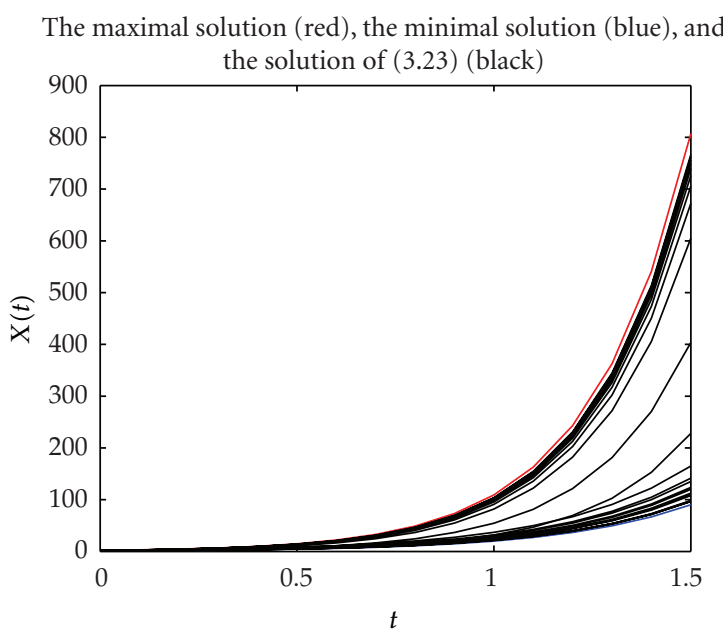

Figure 1: The minimal and maximal solutions of (3.23).

By using Corollary 3.5, then we get $X(t)<W^{n+1}(t, \varepsilon)$ on $[0, \pi / 2]$. The uniqueness of maximal solution $\bar{M}(t)$ show that $W^{n+1}(t, \varepsilon)$ tends uniformly to $\bar{M}(t)$ is the maximal solution of (3.23) with

$$
\bar{M}(t)=\lim _{k \rightarrow \infty} W^{(n+1)_{k}}(t, \varepsilon)
$$

Finally, we will prove (b). Similarly, let $0<\varepsilon_{2}<\varepsilon_{1} \leq \varepsilon$, then for each positive integer $n$, we consider

$$
\begin{gathered}
D_{H} V^{n+1}(t)=3 V^{n}(t)-\frac{\varepsilon}{n}, \quad V^{n+1}(0)=X_{0}-\frac{\varepsilon}{n} \\
V(t)=X_{0} e^{3 t}-\varepsilon .
\end{gathered}
$$

Because $V^{n+1}\left(0, \varepsilon_{1}\right) \leq V^{n+1}\left(0, \varepsilon_{2}\right)$ and $D_{H} V^{n+1}\left(t, \varepsilon_{2}\right) \leq 3 V^{n}(t)-\varepsilon_{2} / n$, otherwise $D_{H} V^{n+1}\left(t, \varepsilon_{1}\right) \leq$ $3 V^{n}(t)-\varepsilon_{2} / n$, hence $D_{H} V^{n+1}\left(t, \varepsilon_{1}\right) \leq D_{H} V^{n+1}\left(t, \varepsilon_{2}\right)$. By using Corollary 3.5 , to get $V^{n+1}\left(t, \varepsilon_{1}\right) \leq$ $V^{n+1}\left(t, \varepsilon_{2}\right)$ for all $t \in[0, \pi / 2]$ and $\varepsilon_{2}<\varepsilon_{1}$. On the other $V^{n}(t) \leq V^{n+1}(t)$ with $\varepsilon$ fixed.

Since the family of functions $\left\{V^{n+1}(t, \varepsilon)\right\}$ is equicontinuous and uniformly bounded on $[0, \pi / 2]$, it follow by Ascoli-Arzela theorem (see [4]) that there exists a decreasing sequence $\left\{\varepsilon /(n+1)_{k}\right\} \stackrel{k \rightarrow \infty}{\longrightarrow} 0$ and uniform limit $\underline{M}(t)=\lim _{k \rightarrow \infty} V^{(n+1)_{k}}(t, \varepsilon)$ exits on $[0, \pi / 2]$. Obviously $M(t)=X_{0} \cdot e^{3 t}$, the uniform continuity of $F$ implies that $F\left(t, V^{(n+1)_{k}}(t, \varepsilon), H\left(t, V^{(n+1) \overline{k_{k}}}(t, \varepsilon)\right)\right)$ tends uniformly to $F(t, \underline{M}(t), H(t, \underline{M}(t)))$ as $k \rightarrow \infty$, and thus

$$
V^{(n+1)_{k}}(t, \varepsilon)=X_{0}+\frac{\varepsilon}{n_{k}}+\int_{0}^{\pi / 2}\left(3 V^{(n+1)_{k}}(s, \varepsilon)\right) d s
$$

which in turn yields that the limit $\underline{M}(t)$ is a solution of $(1)$ on $[0, \pi / 2]$. 
Next we will show that $M(t)$ is a required minimal solution of $(3.23)$ on $[0, \pi / 2]$. For this purpose, we observe that $\bar{X}(0)=X_{0}>X_{0}-\varepsilon / n=V^{n+1}(0, \varepsilon)$ and $F$ is nondecreasing, hence we get

$$
D_{H} X(\mathrm{t})>3 X(t)+\sin (t) X(t)-\frac{\varepsilon}{n} \geq 3 V^{n}(t, \varepsilon)+\sin (t) V^{n}(t, \varepsilon)-\frac{\varepsilon}{n} \geq D_{H} V^{n+1}(t, \varepsilon) .
$$

By using Corollary 3.5, then we get $V^{n+1}(t, \varepsilon)<X(t)$ on $[0, \pi / 2]$. The uniqueness of minimal solution $\underline{M}(t)$ show that $V^{n+1}(t, \varepsilon)$ tends uniformly to $\underline{M}(t)$ is the minimal solution of (3.23) with

$$
\underline{M}(t)=\lim _{k \rightarrow \infty} V^{(n+1)_{k}}(t, \varepsilon)
$$

Based on (3.25) combining (3.28), (3.29) and (3.32), we will solve the minimal and maximal solutions of (3.23). Its graphical representation can be seen in Figure 1.

\section{Acknowledgments}

The authors gratefully acknowledge the referees for their careful reading and many valuable remarks which improved the presentation of the paper.

\section{References}

[1] V. Lakshmikantham, T. G. Bhaskar, and J. V. Devi, Theory of Set Differential Equations in Metric Spaces, Cambridge Scientific Publishers, Cambridge, UK, 2006.

[2] B. G. Pachpatte, Integral and Finite Difference Inequalities and Applications, vol. 205 of North-Holland Mathematics Studies, Elsevier, Oxford, UK, 2006.

[3] S. Hong, "Differentiability of multivalued functions on time scales and applications to multivalued dynamic equations," Nonlinear Analysis, Theory, Methods and Applications, vol. 71, no. 9, pp. 3622-3637, 2009.

[4] S. Hong and J. Liu, "Phase spaces and periodic solutions of set functional dynamic equations with infinite delay," Nonlinear Analysis, Theory, Methods and Applications, vol. 74, no. 9, pp. 2966-2984, 2011.

[5] J. V. Devi, "Generalized monotone iterative technique for set differential equations involving causal operators with memory," International Journal of Advances in Engineering Sciences and Applied Mathematics, vol. 3, no. 1-4, pp. 74-83, 2011.

[6] N. D. Phu and T. T. Tung, "Some results on sheaf-solutions of sheaf set control problems," Nonlinear Analysis, Theory, Methods and Applications, vol. 67, no. 5, pp. 1309-1315, 2007. 


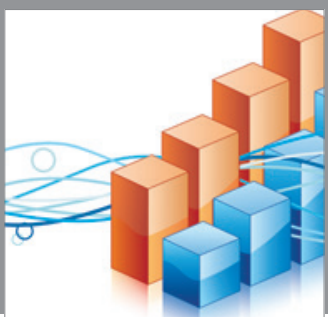

Advances in

Operations Research

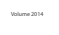

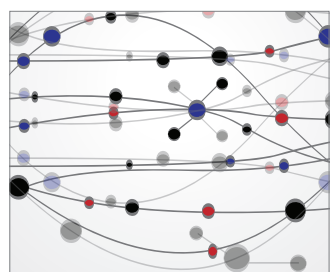

\section{The Scientific} World Journal
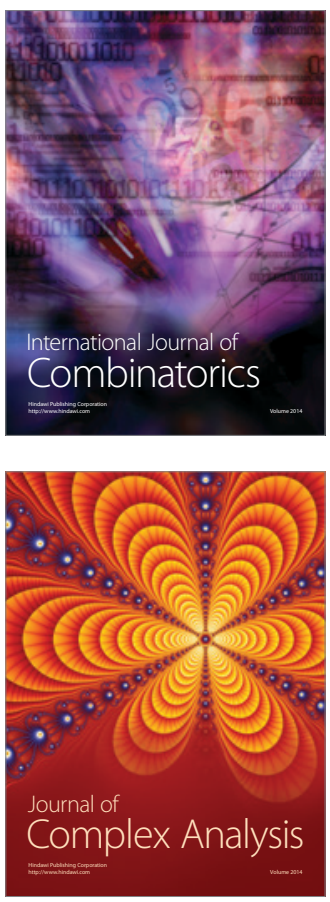

International Journal of

Mathematics and

Mathematical

Sciences
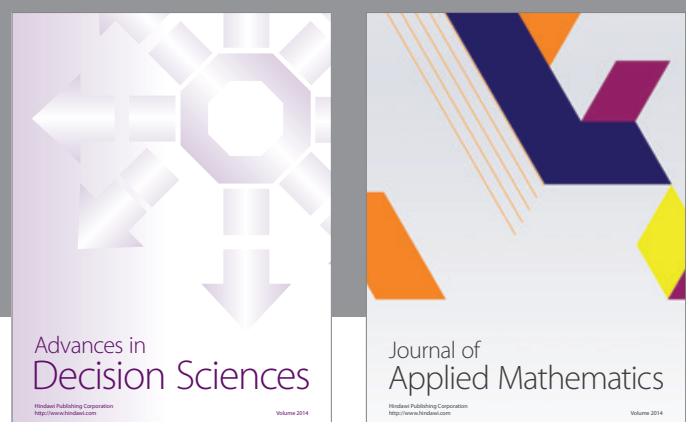

Journal of

Applied Mathematics
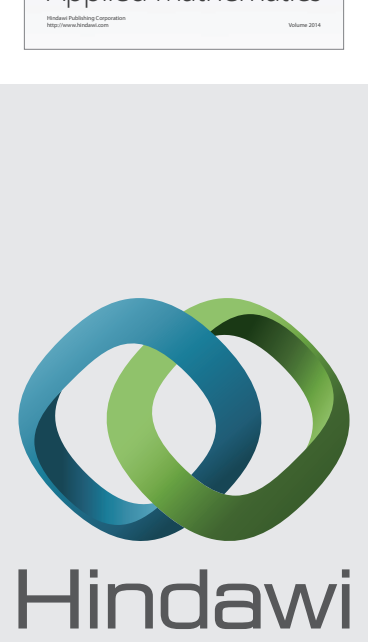

Submit your manuscripts at http://www.hindawi.com
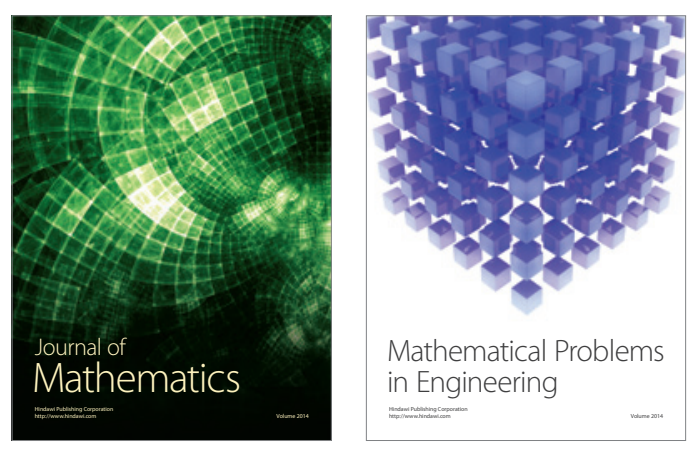

Mathematical Problems in Engineering
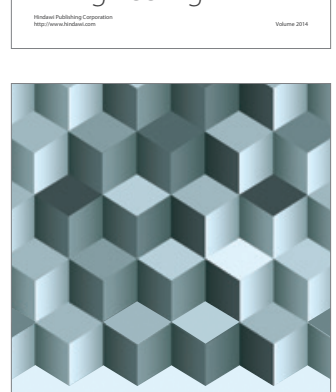

Journal of

Function Spaces
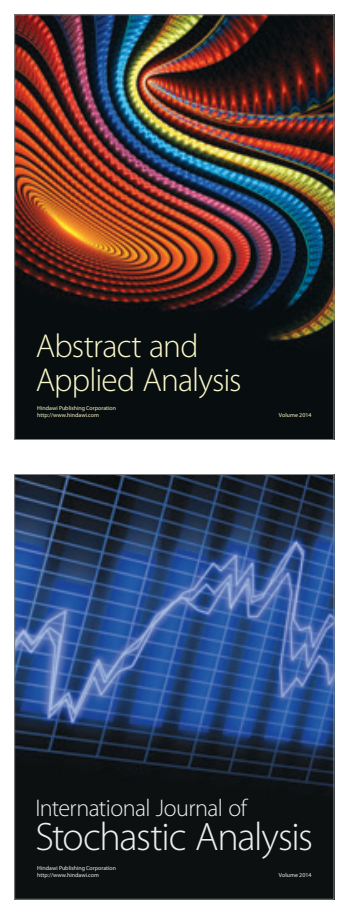

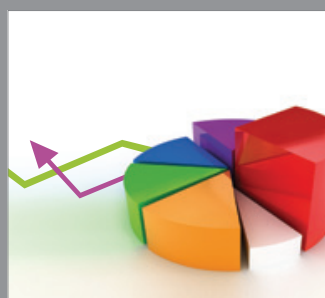

ournal of

Probability and Statistics

Promensencen
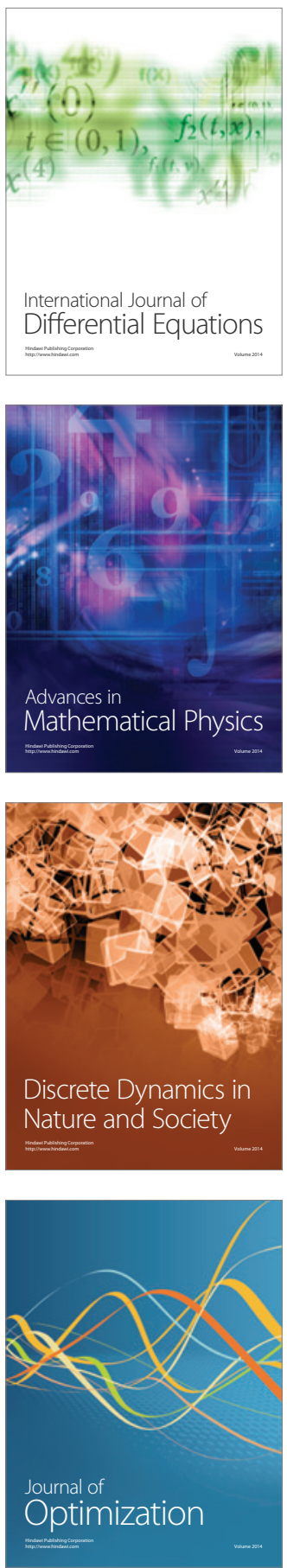